\title{
Designing Customized 3D Printed Models for Surgical Planning in Repair of Congenital Heart Defects
}

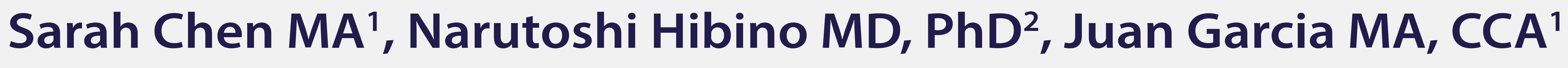

'Johns Hopkins School of Medicine, Department of Art as Applied to Medicine; ${ }^{2} J o h n s$ Hopkins Hospital, Division of Cardiac Surgery

\section{Abstract}

Advances in diagnostic imaging and 3D printing technology have enabled the creation of patient-specific models. This research established a workflow for creating 3D printed congenital heart defect (CHD) models, focusing specifically on creating aortic arch models optimized for surgical planning and simulation for hypoplastic left heart syndrome (HLHS) stage I palliation. Novel methods for creating $\mathrm{CT}$ as well as 3D ultrasound and 3D fetal ultrasound derived prints were explored.

\section{Introduction}

Hypoplastic left heart syndrome (HLHS) is a condition in which the left side of the heart is severely underdeveloped, and one of the most fatal CHDs if left untreated' Surgical palliation consists of three separate, staged surgeries. Aortic arch morphology following the stage I Norwood procedure has been found to be especially critical in determining cardiac function and outcome ${ }^{2}$.

Pre-surgical planning is crucia

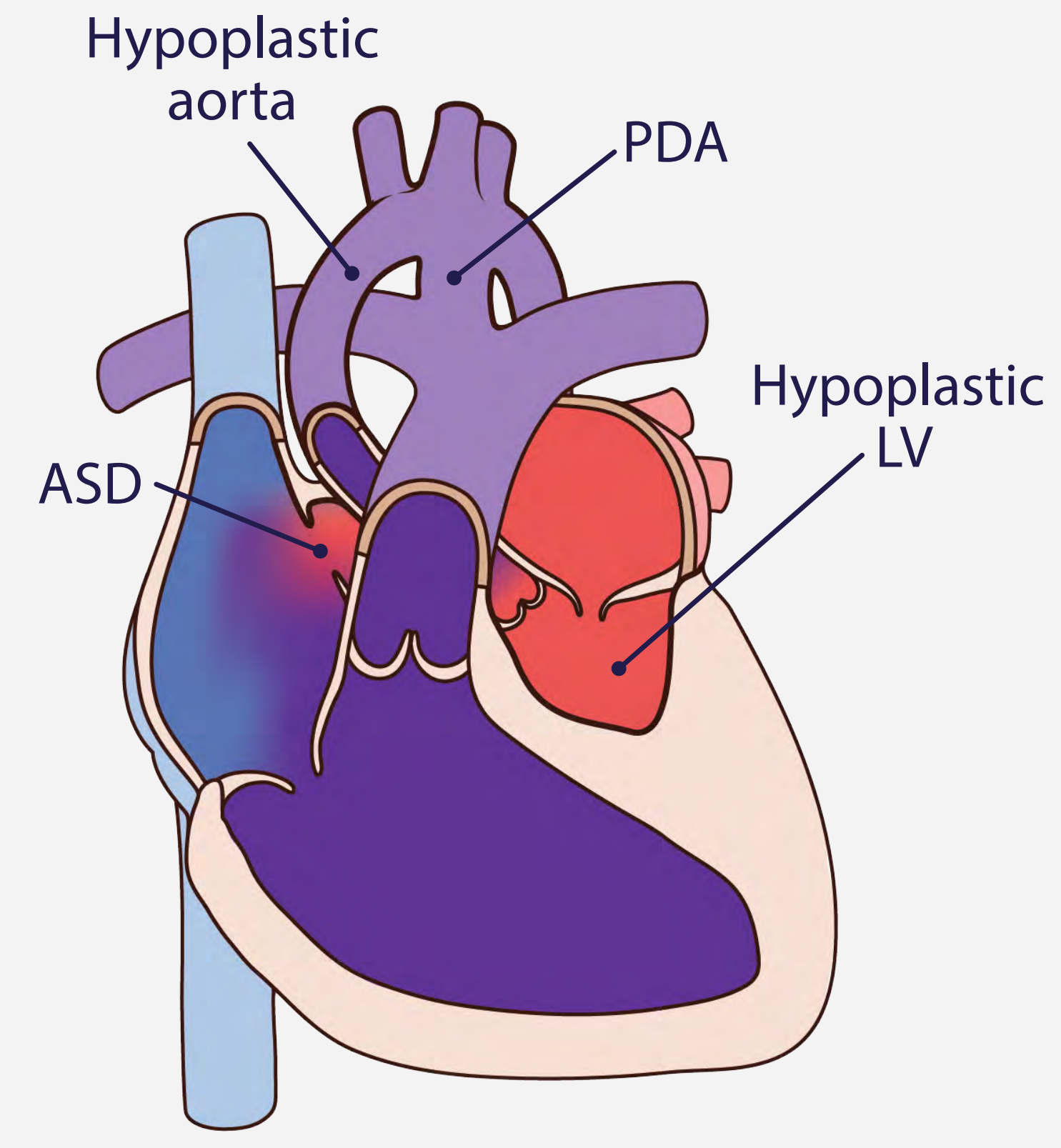

Fig 1. HLHS anatomy so that surgeons can know what to expect before going to the $\mathrm{OR}$, and 3D printed cardiac models created for surgical planning and simulation can enhance surgical precision and reduce OR/anesthesia/bypass times and post-op complications.
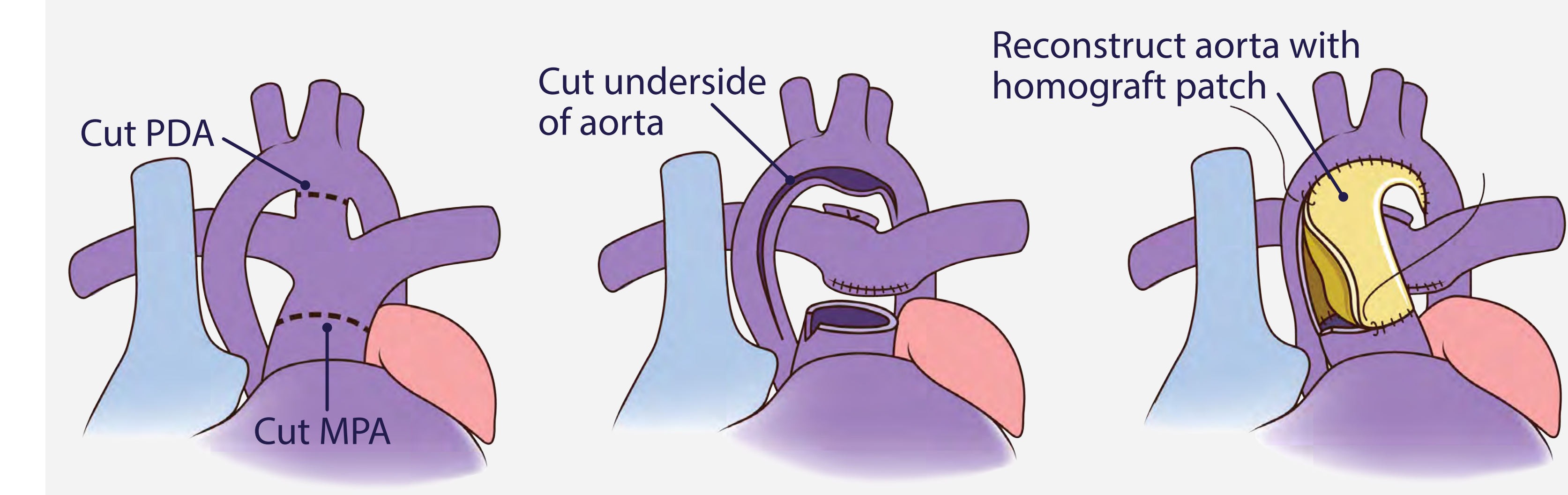

Fig 2. Aortic arch reconstruction using homograft during Norwood procedure

\section{Objectives}

Intended outcomes were to create:

-3D print: pre-operative HLHS aortic arch model

-3D print: post-operative HLHS aortic arch mode

-3D print: custom designed homograft patch

-3D prints: proof-of-concept models from 3D ultrasound

- Documented workflow

\section{Materials \& Methods}

Materialise Mimics segmentation software was used to isolate aortic arch anatomy from HLHS CT scans. Autodesk Meshmixer was used to optimize the exported segmented model, and to design the shape of the homograft patch customized to allow for the desired post-operative result. The models were printed on a Stratasys Connex3 Objet260 PolyJet printer in Tango Plus, a clear, flexible material to allow surgical suturing to assess the homograft patch.
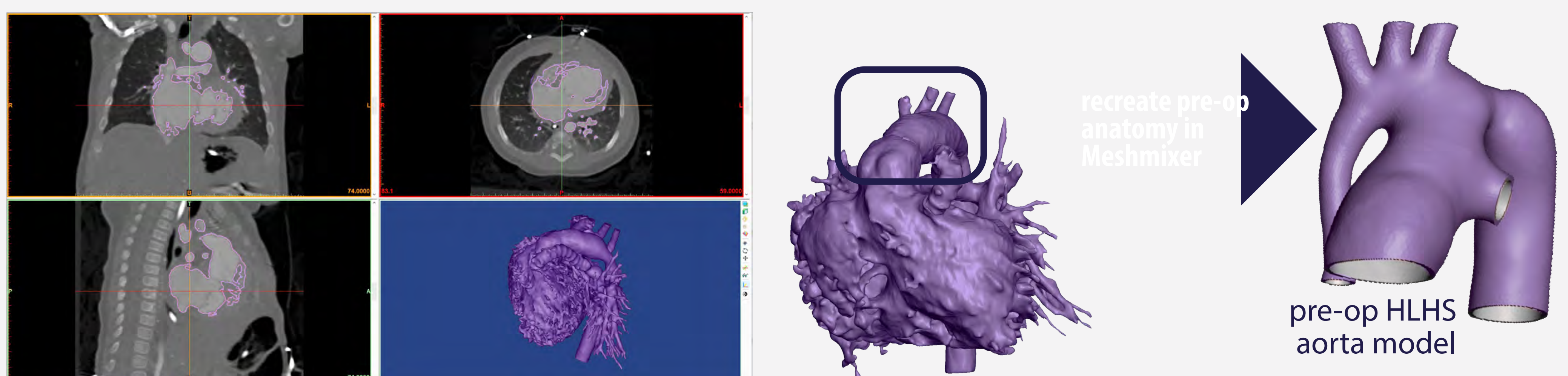

Fig 3. Left: segmentation of HLHS CT using Mimics. Right: digital manipulation of HLHS model (CT$$
\text { M }
$$

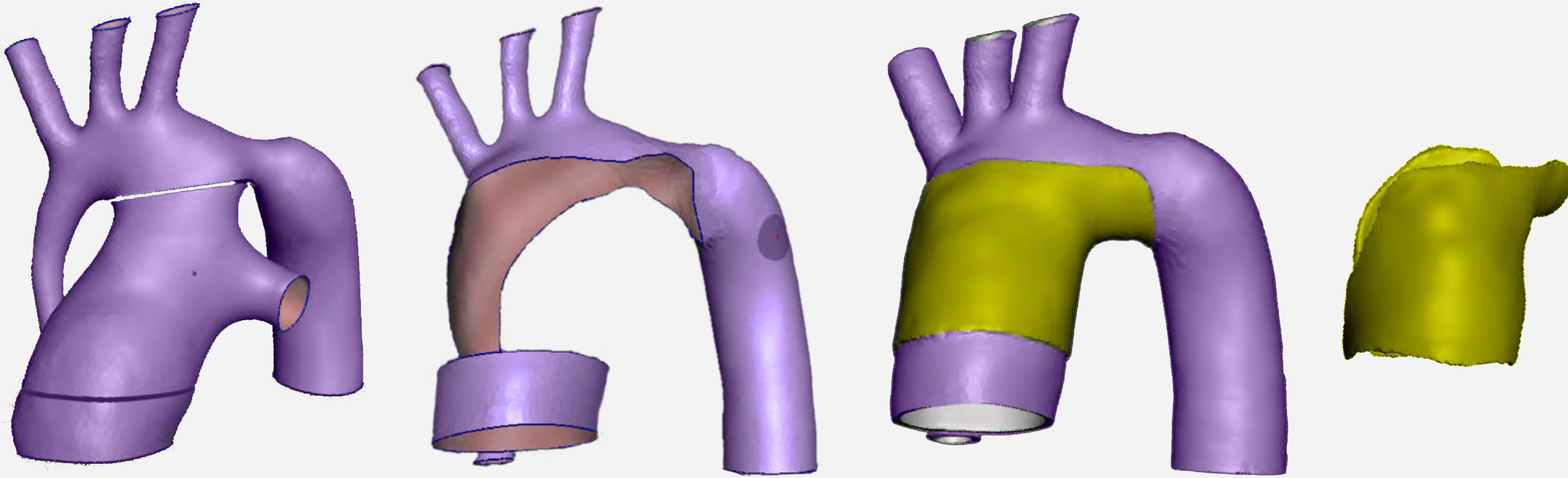

Surgical Simulation: The 3D printed models were used to simulate the Norwood surgery. The customized homograft patch was used to repair the pre-op HLHS model and assessed for fit. Supports were designed to hold the model in place during the simulated operation.

Ultrasound-derived 3D Prints: Additional proof-of-concept 3D prints were created from normal 3D aortic arch ultrasound as well as 3D fetal cardiac ultrasound data, to utilize a safe, cost-effective imaging modality without harmful radiation or anesthesia in vulnerable pediatric patients. Segmentation of 3D ultrasound data was possible using Materialise Mimics Research Edition.

3D ultrasound imaging of the aortic arch was obtained with a Philips EPIQ 7 scanner and exported as a Cartesian DICOM file to create an aortic arch model. A fetal heart model was created from a 3D fetal ultrasound data set of a 27-week old fetus that was obtained from a GE Voluson E10 scanner and exported as a Cartesian .vol file. Models were cleaned up in Meshmixer and 3D printed.

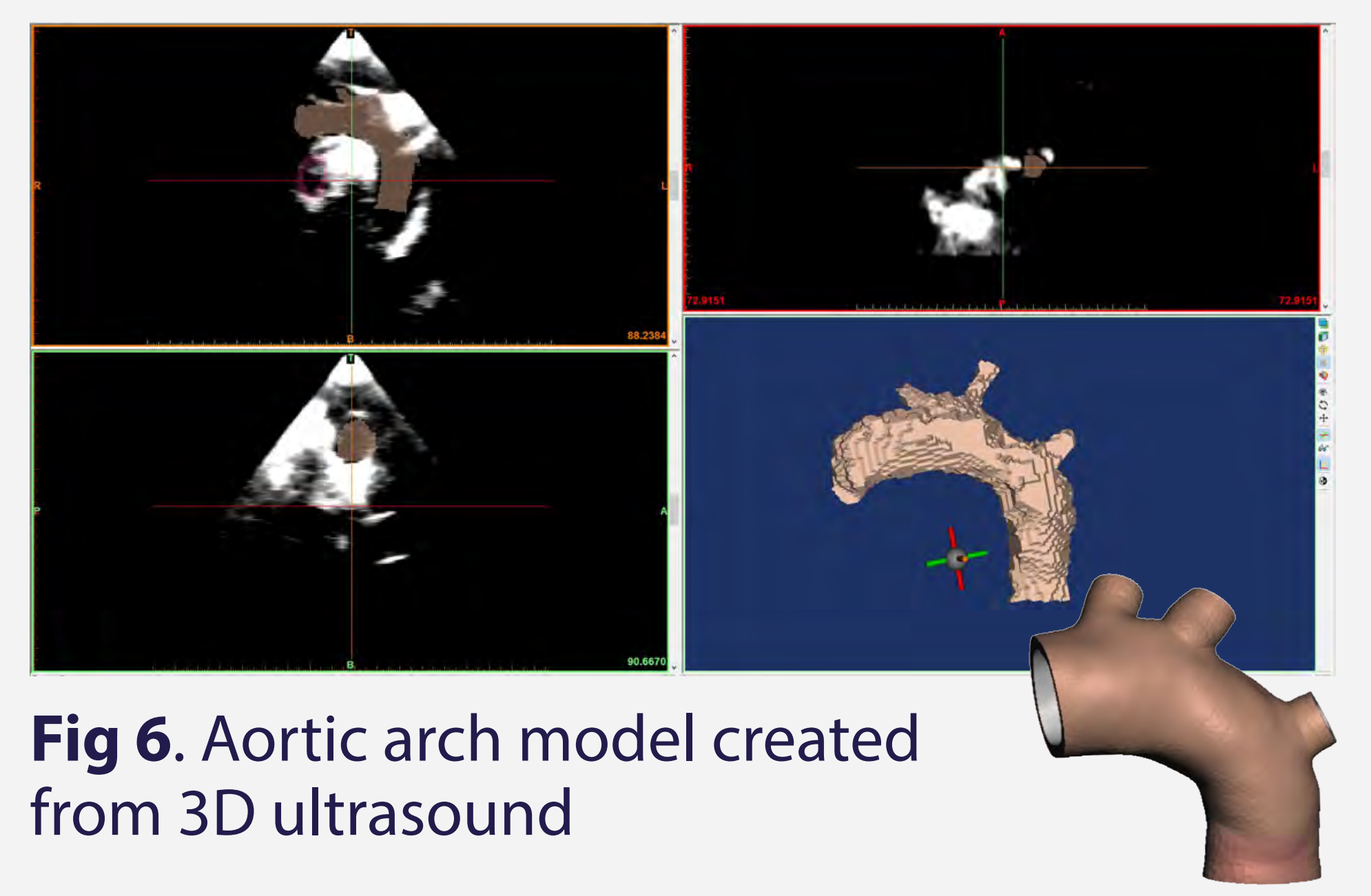

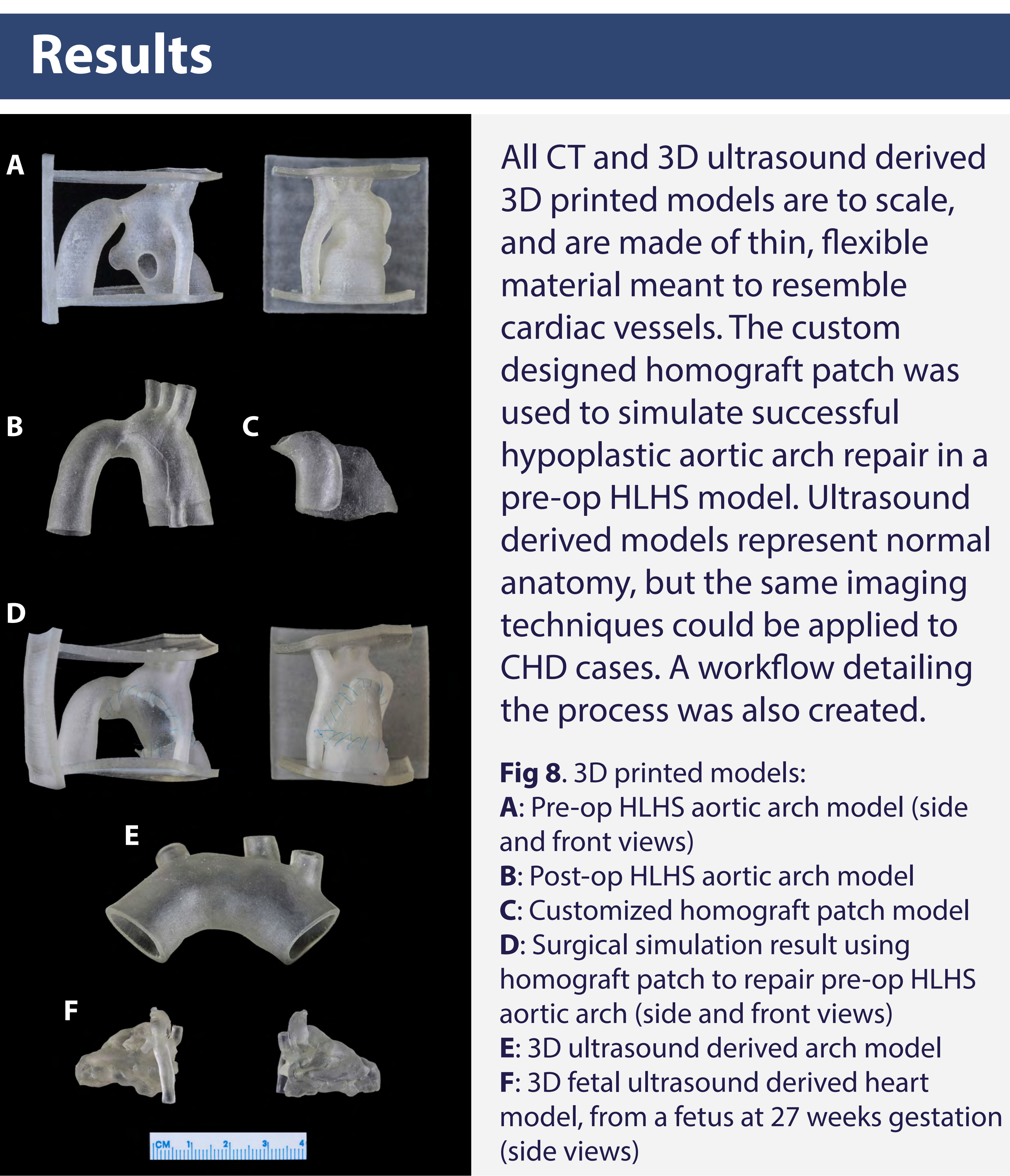

Discussion \& Conclusions

The established workflow promotes a novel method for producing ultrasound-derived patient-specific 3D printed cardiac models, as well as a method to custom design surgical patches, which have the potential to improve treatment planning and patient outcomes. 3D printed models derived from 3D ultrasound provide a safer alternative to $C T$ or MRI based prints, while creating models from fetal imaging could allow surgeons more time for pre-procedural planning.

Medical 3D printing is a powerful tool that can revolutionize personalized, precision medicine through the design and implementation of customized surgical guides and patches.

\section{References}

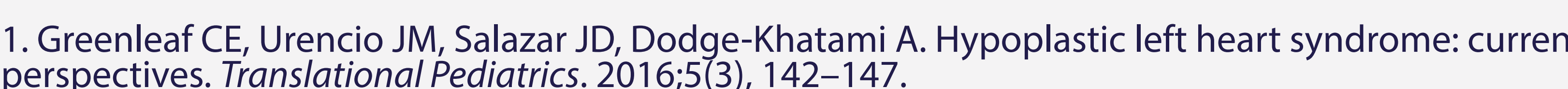

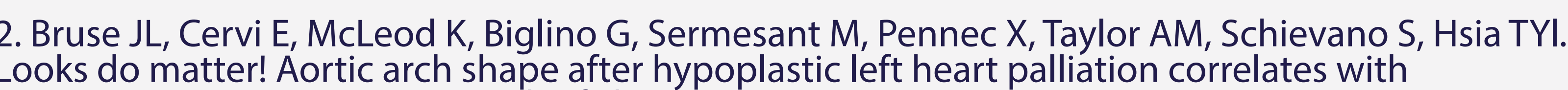

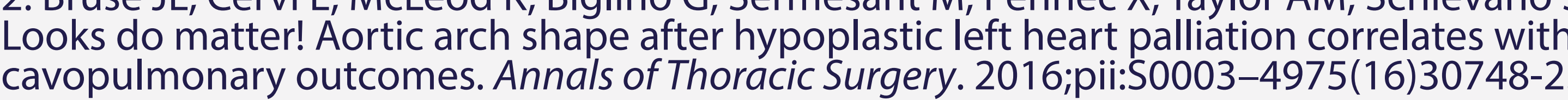
3. Giannopoulos AA, Mitsouras D, Yoo SJ, Liu PP, Chatzizisisis YS, Rybicki F. A. Applications of $3 \mathrm{D}$
printing in cardiovascular diseases. Nature Reviews Cardiology. 2016; $13(2), 701-718$. 